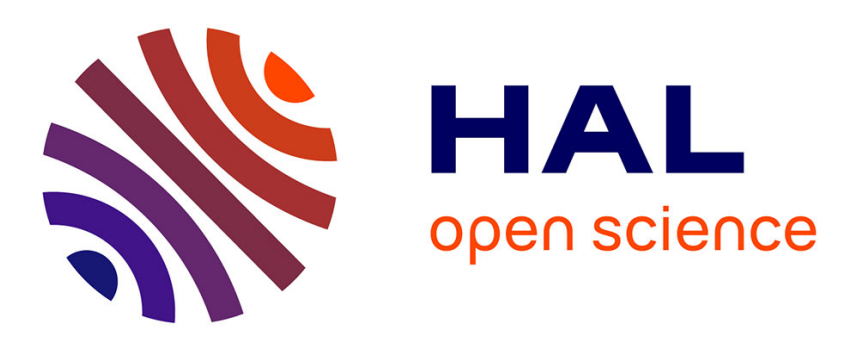

\title{
Some characteristics of Josephson junctions as radiation detectors
}

\author{
Yu. Ya. Divin, F. Ya. Nad, O. Yu. Polyanskii, A.F. Volkov
}

\section{To cite this version:}

Yu. Ya. Divin, F. Ya. Nad, O. Yu. Polyanskii, A.F. Volkov. Some characteristics of Josephson junctions as radiation detectors. Revue de Physique Appliquée, 1974, 9 (1), pp.125-130. 10.1051/rphysap:0197400901012500 . jpa-00243721

\section{HAL Id: jpa-00243721 https://hal.science/jpa-00243721}

Submitted on 1 Jan 1974

HAL is a multi-disciplinary open access archive for the deposit and dissemination of scientific research documents, whether they are published or not. The documents may come from teaching and research institutions in France or abroad, or from public or private research centers.
L'archive ouverte pluridisciplinaire HAL, est destinée au dépôt et à la diffusion de documents scientifiques de niveau recherche, publiés ou non, émanant des établissements d'enseignement et de recherche français ou étrangers, des laboratoires publics ou privés. 


\title{
SOME CHARACTERISTICS OF JOSEPHSON JUNCTIONS AS RADIATION DETECTORS
}

\author{
Yu. Ya. DIVIN, F. Ya. NAD', O. Yu. POLYANSKII and A. F. VOLKOV \\ Institute of Radioengineering and Electronics \\ of the Academy of Sciences of the USSR, Moscow, USSR
}

\begin{abstract}
Résumé. - On présente les calculs, de la caractéristique $V-I$ d'une jonction Josephson shuntée par une résistance, de son impédance en haute fréquence et de sa réponse à un petit signal externe. On donne les courbes expérimentales obtenues dans le domaine de longueurs d'onde de 5 à $30 \mathrm{~cm}$ et on précise les conditions optimales de fonctionnement pour les contacts à pointes et les microponts.

Abstract. - The $V-I$ characteristic of Josephson junction with an external parallel admittance, the high frequency impedance and high frequency response of a Josephson junction to small external signal are calculated by analytical method. The experimentally obtained spectral response curves for the superconducting point contacts in the frequency range $5-30 \mathrm{~cm}^{-1}$ are presented. The condition need for achieving high sensitivity of superconducting point contacts and superconducting bridges are determined.
\end{abstract}

It is known that weak links (Josephson junctions) may be used for realizing various types of radiation detectors in the millimeter and submillimeter frequency range, namely for broadband [1]-[3] and narrow-band detectors [4], and also for frequency mixing and conversion [5]-[7].

Previously, the effects of radiation on the voltagecurrent characteristic (VIC), of a Josephson junction and the behavior of a junction in a microwave field were investigated both theoretically and experimentally [1], [8], [9]. However, some questions which are important from the point of view of the development of a detector remained unclear. It is worth while to note also that many of these results, for example, concerning the behavior of a Josephson junction in parallel with an external impedance were derived by numerical methods [13].

In the first section of this paper, the results of an analytical calculation of the VIC of a Josephson junction with an external parallel admittance $Y(\omega)$ and the calculation of the junction impedance are presented. The expression for responsivity of Josephson junction to external radiation is obtained and in the second section of this paper the experimental spectral response curves in the frequency range $5-30 \mathrm{~cm}^{-1}$ are given. Finally, in the third section, the conditions needed for achieving high sensitivity of superconducting bridge Josephson junctions are determined.

1. Effect of an external perturbation on a Josephson junction. Theory. - We shall proceed from the equation for the phase difference of the order parameter for a Josephson junction with a quasiparticle current, as is present in a superconducting point contact, a superconducting bridge, etc. In dimensionless variables this equation is

$$
\dot{\varphi}_{0}+\sin \varphi_{0}=j_{0} .
$$

Here $j_{0}=I / I_{\mathrm{c}}, I$ is the constant dc current through the junction, $I_{\mathrm{c}}$ is the critical Josephson current, $\varphi_{0}$ is the phase difference of the order parameter, the dot denotes differentiation with respect to dimensionless time $\tau=t / t_{0}$, where $t$ is the time, $t_{0}=\hbar / 2 \mathrm{e} I_{\mathrm{c}} R$, and $R$ is the junction resistance in the normal state. This equation describes the autonomous Josephson junction and has the analytic solution corresponding to self oscillations of the voltage across the junction,

$$
\begin{aligned}
\dot{\varphi}_{0}(\tau) & =\frac{v^{2}}{j_{0}-\cos v \tau}=\sum_{-\infty}^{+\infty} a_{\mathrm{n}} \mathrm{e}^{i n v \tau}, \\
a_{\mathrm{n}} & =v\left(j_{0}-v\right)^{|n|} .
\end{aligned}
$$

The relation between the current and the average dimensionless voltage $v$ is given by the well known expression $j_{0}=\sqrt{1+v^{2}}$. If the junction is connected to a parallel external impedance or is affected by external radiation, then a perturbation term $f(\tau)$ must be added to the right-hand side of eq. (1), giving

$$
\dot{\varphi}+\sin \varphi=j+f(\tau) \text {. }
$$

In the general case it is impossible to solve the eq. (3) analytically. However, if we assume that the pertur- 
bation is small, then an approximate solution may be obtained by expanding $\varphi$ in a series

$$
\varphi=\varphi_{0}+\varphi_{1}+\varphi_{2}+\cdots,
$$

where the expansion is carried out in powers of small perturbation, and $\varphi_{0}$ is given by expression (2). The current $j$ is determined by series $j=j_{0}+j_{1}+j_{2}+\cdots$, where $j_{1}, j_{2}, \ldots$ are the time independent corrections to the VIC due to this perturbation. The first order correction $\varphi_{1}$ is obtained from linearized eq. (3) and has the form

$$
\varphi_{1}=\dot{\varphi}_{0}(\tau)\left\{\int_{0}^{\tau} \dot{\varphi}_{0}^{-1}\left(\tau^{\prime}\right)\left[j_{1}+f\left(\tau^{\prime}\right)\right] \mathrm{d} \tau^{\prime}\right\} .
$$

The iterative procedure carried out by us is correct when $\varphi_{1} \ll 1$. In order to fulfill this condition it is necessary that the time divergent terms should be equal to zero. Thus we obtain the first order correction to the VIC

$$
j_{1}=-\lim _{\tau \rightarrow \infty} \frac{\int_{0}^{\tau} f\left(\tau^{\prime}\right) \dot{\varphi}_{0}^{-1}\left(\tau^{\prime}\right) \mathrm{d} \tau^{\prime}}{\int_{0}^{\tau} \dot{\varphi}_{0}^{-1}\left(\tau^{\prime}\right) \mathrm{d} \tau^{\prime}} .
$$

Note also that with some $f(\tau)$ and $j, \varphi_{1}$ may involve singular terms. In this case the solution may be obtained by imposing further limitations on $j$ [9].

At first let us consider the Josephson junction connected in parallel with an admittance $Y(\omega)$ [16]. Then the perturbation $f(\tau)$ is

$$
f(\tau)=-\int_{0}^{\infty} \gamma\left(\tau^{\prime}\right) \dot{\varphi}\left(\tau-\tau^{\prime}\right) \mathrm{d} \tau^{\prime},
$$

where $\gamma(\tau)$ is the reduced term related to the admittance $Y(\omega)$ by Fourier transformation

$$
\gamma(\tau)=\frac{R}{\omega_{0}} \int_{-\infty}^{\infty} Y(\omega) \mathrm{e}^{i \omega \tau} \mathrm{d} \omega .
$$

In this case the perturbation is small, if $\left[Y\left(\omega_{0}\right)\right]^{-1} \gg R$, where $\omega_{0}=2 \mathrm{e} I_{\mathrm{c}} R / \hbar$, the characteristic frequency of Josephson oscillations. Then according to eq. (5) the correction to the VIC is

$$
j_{1}=\sigma(0) v+\frac{v\left(v-\sqrt{v^{2}+1}\right)}{\sqrt{v^{2}+1}} \operatorname{Re} \sigma(v) .
$$

Here we used the definition of $\gamma(\tau)$ given by eq. (7) and the zero order solution of eq. (2);

$$
\sigma(n v)=Y\left(n v \omega_{0}\right) R
$$

is the reduced admittance at the frequency $\omega=n v \omega_{0}$. It may be seen from eq. (8), for example, that if $\sigma(0)=0$ then the correction to the $\mathrm{VIC} j_{1}$ divided by $a_{1}(v) / j_{0}$ determines the dispersion of the real part of the admittance $\sigma(v)$.

Hence, the measurement of the VIC of a Josephson junction makes it possible, in principle, to determine the spectral distribution of losses in the system under consideration. Experimentally, point contact were used for spectroscopic studies by A. Silver and J. Zimmerman [10].

Consider now a Josephson junction influenced by high frequency monochromatic radiation at a frequency $\omega_{1}$. In this case the equation (3) has the form

$$
\dot{\varphi}+\sin \varphi=j+j_{\sim} \sin (\Omega \tau) \text {. }
$$

Here $j_{\sim}=I_{1} / I_{\mathrm{c}}, \Omega=\omega_{1} t_{0}, j_{\sim} \ll 1$. The value of $\varphi_{1}$ is determined by expression (4), where in this case, as can be easily proved, $j_{1}=0$.

Of all the oscillation frequency components present in the junction, only two frequencies are of interest to us in the case under consideration, namely forced oscillations $v_{1}$, at external frequency $\Omega$, and the component at the difference frequency $(v-\Omega)$ denoted by $v_{2}$. Hence in expanding $\dot{\varphi}$ we restrict ourselves to the terms with $n=0,1,2$ only, because the amplitudes of these components are independent on the terms with larger $n$. Extracting the terms corresponding to the oscillations at frequencies $\Omega$ and $(v-\Omega)$ we get

$$
\begin{aligned}
& v_{1}=j_{\sim} \frac{\Omega}{v^{2}}\left[\frac{a_{0} j_{0}}{\Omega}+\frac{a_{1} \Omega}{v^{2}-\Omega^{2}}\right] \\
& v_{2}=j_{\sim} \frac{v-\Omega}{v^{2}}\left[\frac{a_{1}}{\Omega}-\frac{j_{0}}{2(\Omega+v)}+\frac{a_{2}}{2(v-\Omega)}\right] .
\end{aligned}
$$

These solutions are correct at any $v$ provided

$$
|v-\Omega| \gg j_{\sim} / \Omega
$$

(this condition provides that $\varphi_{1}$ is small), i. e. at all points of VIC except the vicinity of the current step $v \simeq \Omega$ and $\Omega=0$

It is convenient to analyse the interaction of the electromagnetic wave oscillator with the Josephson junction by means of the equivalent circuit shown in figure 1 . The case just considered corresponds to the condition of infinite $Z_{2}\left(\omega_{2}\right)$, i. e. the junction is not shunted at the frequency $\omega_{2}$. In this case, however, the voltage at the difference frequency $\omega_{2}$ develops across the junction also, as a result of the interaction of the external oscillations at frequency $\omega_{1}$ with the self-oscillation in the junction. Now, if the junction is shunted at the frequency $\omega_{2}$ (for example it is coupled to the suitable waveguide with impedance $Z_{2}\left(\omega_{2}\right)$ ).

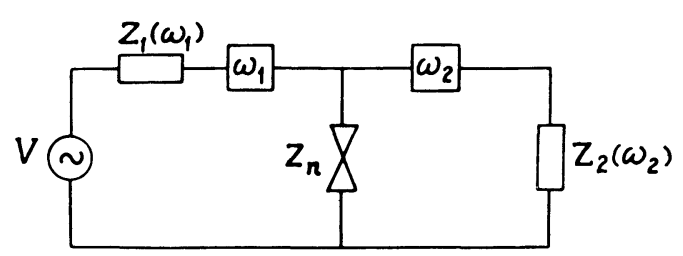

FIG. 1. - Equivalent circuit of a Josephson junction, connected to two waveguides and a generator. $V$ is the voltage source ; $Z_{1}\left(\omega_{1}\right)$ and $Z_{2}\left(\omega_{2}\right)$ are the equivalent impedances of the input and output waveguides, $Z_{\mathrm{n}}$ is the Josephson junction impedance ; $\omega_{1}$ and $\omega_{2}$ are ideal filters, passing only one frequency 
then a current $I_{2}$ at the difference frequency $\omega_{2}$ begins to flow as well.

Assuming $I_{1}, I_{2} \ll I$ one may suggest, that these currents do not change the characteristics of junction (characteristics of junction are changed only by the action of the current $I$ ) and they are independent from each other, i. e. one can use the principle of superposition. Using this circumstance, one can show, that in the presence of two circuits, coupled with the Josephson junction at frequencies $\omega_{1}$ and $\omega_{2}$, the complex amplitudes of the voltage $V_{1}\left(\omega_{1}\right)$ and $V_{2}\left(\omega_{2}\right)$ are given by

$$
\begin{aligned}
& \dot{V_{1}}\left(\omega_{1}\right)=Z_{\mathrm{n}}\left(\omega_{1}\right) \dot{I}_{1}+Z_{\mathrm{c}_{1}} \dot{I}_{2}^{*} \\
& \dot{V}_{2}\left(\omega_{2}\right)=Z_{\mathrm{n}}\left(\omega_{2}\right) \dot{I}_{2}+Z_{\mathrm{c}_{2}} \dot{I}_{1}^{*}
\end{aligned}
$$

(the symbol * denotes the complex conjugate), where $Z_{\mathrm{n}}\left(\omega_{1}\right)=\dot{V}_{1} / \dot{I}_{1}$ is the impedance of the junction at $\omega_{1}$, without current at $\omega_{2}\left(\dot{I}_{2}=0\right) ; Z_{\mathrm{n}}\left(\omega_{2}\right)=\dot{V}_{2} / \dot{I}_{2}$ is the impedance of the junction at $\omega_{2}$, without current at $\omega_{1}\left(I_{1}=0\right) ; Z_{\mathrm{c}_{1}}=\dot{V}_{1} / \dot{I}_{1}^{*}$ with $\dot{I}_{1}=0$ and $Z_{\mathrm{c}_{2}}=\dot{V}_{2} / \dot{I}_{1}^{*}$ with $\dot{I}_{2}=0$ are the coupled impedances caused by the interaction of the current $I_{2}$ with $V_{1}$ and $I_{2}$ with $V_{2}$ due to the frequency conversion. From eq. (10) and (11) we obtain the equation for the impedance of the Josephson junction :

$$
\begin{aligned}
Z_{\mathrm{n}}\left(\omega_{1}\right) & =\frac{\Omega}{v^{2}}\left[\frac{a_{0} j_{0}}{\Omega}+\frac{a_{1} \Omega}{v^{2}-\Omega^{2}}\right] \\
Z_{\mathrm{n}}\left(\omega_{2}\right) & =\frac{v-\Omega}{v^{2}}\left[\frac{a_{0} j_{0}}{v-\Omega}+\frac{a_{1}(v-\Omega)}{\Omega(2 v-\Omega)}\right] \\
Z_{\mathrm{c}_{1}} & =i \frac{v-\Omega}{v^{2}}\left[\frac{a_{0}}{2(v-\Omega)}+\frac{a_{1} j_{0}}{\Omega}-\frac{a_{2}}{2(v+\Omega)}\right] \\
Z_{\mathrm{c}_{2}} & =i \frac{\Omega}{v^{2}}\left[\frac{a_{0}}{2 \Omega}+\frac{a_{1} j_{0}}{v-\Omega}-\frac{a_{2}}{2(2 v-\Omega)}\right]
\end{aligned}
$$

Using this matrix of impedances one can determine the conversion gain $G$ of the Josephson junction, ascertain the conditions for regeneration, the frequency band, and finally the noise temperature of the converter [11]. For example, the conversion gain in the presence of the matching in the limit of low voltage $(v \ll 1)$ and low frequency $(v \gg \Omega)$ has the simple form,

$$
G_{\mathrm{opt}}=\frac{1+2 \omega_{2} / \omega_{1}}{1+2 \omega_{1} / \omega_{2}}
$$

This results in $G>1$, when $\omega_{2}>\omega_{1}$ and $G<1$, when $\omega_{2}<\omega_{1}$, i. e. it is possible to obtain amplification for frequency up-conversion. The noise characteristics of the frequency converter based on a Josephson junction may be estimated using the proper equivalent noise temperature. Taking into account the data of reference [12] we deduce that the noise temperature is equal to $50 \mathrm{~K}$ for a resistive Josephson junction with normal resistance $R$ being equal to several ohms and for the frequency conversion from $100 \mathrm{GHz}$ to $10 \mathrm{GHz}$.

For a current-driven Josephson junction the dc voltage response $\Delta v$ to an external high-frequency signal is determined by the change in the VIC $\Delta j=j-j_{0}$. As was noted above, the first-order correction to the VIC $j_{1}$ is equal to zero. Thus to determine this response it is necessary to solve eq. (9), by using a second order perturbation approach.

Continuing the iterative procedure on eq. (9) we obtain for $\varphi_{2}$ :

$$
\begin{aligned}
\varphi_{2}=\dot{\varphi}_{0} & \int_{0}^{\tau} \times \\
& \times\left\{\dot{\varphi}_{0}^{-1}\left(\tau^{\prime}\right)\left[j_{2}+\frac{1}{2} \varphi_{1}^{2}\left(\tau^{\prime}\right)\left(j_{0}-\varphi_{0}\left(\tau^{\prime}\right)\right)\right]\right\} \mathrm{d} \tau^{\prime}
\end{aligned}
$$

Demanding that the time-divergent terms in eq. (14) be equal to zero as before, we find the secondorder correction to the VIC

$$
j_{2}=\frac{j_{\sim}^{2}}{4 j_{0}\left(v^{2}-\Omega^{2}\right)} .
$$

Using the relation $\Delta v=\frac{\mathrm{d} v}{\mathrm{~d} j_{0}} \Delta j$, from eq. (15) we obtain the response of a current-driven Josephson junction (in dimensional form) :

$$
\Delta V=\frac{I_{1}^{2} V_{c}^{2} R^{2}}{4 V\left(V^{2}-V_{\omega}^{2}\right)},
$$

where $V_{\mathrm{c}}=I_{\mathrm{c}} R$ is the product of the critical current $I_{\mathrm{c}}$ and the normal resistance $R ; V_{\omega}=\hbar_{\omega} / 2 \mathrm{e}$ is the voltage corresponding to the step on the VIC of the irradiated junction. Analogous results were obtained in reference [2] in another way. The eq. (16) is valid for all voltages except for those very close to $V=0$ and $V=V_{\omega}$.

Using the relation between the absorbed power $W$ and the induced current $I_{1}: W=\frac{1}{2} I_{1}^{2} Z_{\mathrm{n}}(\omega)$, where $Z_{\mathrm{n}}(\omega)$ is the junction impedance given by eq. (12), we find the voltage responsivity $S$. In the high frequency limit $V_{\omega}^{2} \gg V \sqrt{V^{2}+V_{c}^{2}}$

$$
S=\frac{V_{\mathrm{c}}^{2} R}{2 V V_{\omega}^{2}} \text {. }
$$

To estimate the noise-equivalent power $(N E P)$ we use the expression for the mean squared noise voltage from [12]. It is possible to obtain the result that in the high frequency limit for the parts of VIC undisturbed by the noise, the $N E P$ is minimum at the junction voltage $V \simeq k T / \mathrm{e}$ and the value of $N E P$ at this point equals :

$$
N E P=\frac{4 \mathrm{e}^{1 / 2} V_{\omega}^{2}}{V_{c}^{1 / 2} R^{1 / 2}} .
$$


It follows from eq. (17) and (18), that the sensitivity increases with an increase of normal resistance $R$ and characteristic voltage $V_{\mathrm{c}}$. For $\mathrm{Nb}-\mathrm{Nb}$ junctions with $R=5 \Omega$ at $\lambda=0.7 \mathrm{~mm}$ the estimations give the following values of sensitivity : $S=7 \times 10^{4} \mathrm{~V} / \mathrm{W}$,

$$
(N E P)_{\min }=10^{-14} \mathrm{~W} / \mathrm{Hz}^{1 / 2} .
$$

2. Spectral properties. - We have studied experimentally the dependences of $\Delta V(V)$ and $\Delta V(\omega)$ for superconducting point contacts. The measurements were carried out with an adjustable $\mathrm{Nb}-\mathrm{Nb}$ point contacts. Radiation passed down through a light pipe of diameter $20 \mathrm{~mm}$ and was focused by a cone with outer diameter $5 \mathrm{~mm}$. For the investigated contacts, we measured a VIC, voltage dependence of the differential resistance and voltage dependence of the response at $\lambda \sim 5 \mathrm{~mm}$. The contacts with hyperbolic VIC $V=R \sqrt{I^{2}-I_{\mathrm{c}}^{2}}(R$ ranged from few tenths to few tens of ohms) were investigated in most detail.

The recorder plot of a point contact response as a function of dc voltage is shown in figure 2 at three different high frequency power levels. For low voltages $V<V_{\omega}$ the maximum of the response is observed at the point where the differential resistance $R_{\mathrm{D}}$ reachs the peak. We also note a feature at voltage $\sim V_{\omega} / 2$, that is a subharmonic step. In the vicinity of the harmonic step $V_{\omega}=128 \mu \mathrm{V}$ the response changes sign and rises to a larger absolute values than at low voltages. The voltage dependence of the response is in qualitative agreement with theoretically derived eq. (16), which implies that there are two points where $\Delta V$ reaches the maximum : at $V \rightarrow 0$ and $V \rightarrow V_{\omega}$. The noise and external fluctuations

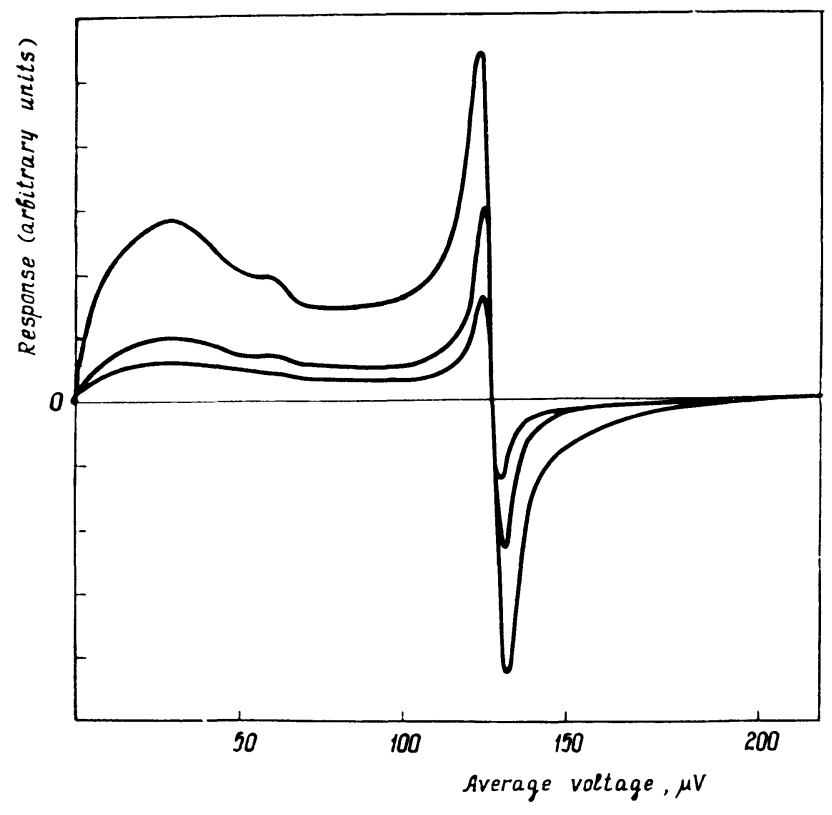

Fig. 2. - Average voltage dependence of the response $\Delta V$ for a $\mathrm{Nb}-\mathrm{Nb}$ point contact at $T=4.2 \mathrm{~K}$ with $R=0.5 \Omega$, $I_{0}=0.34 \mathrm{~mA}$. wash out the singularities and lead to the appearance of finite values of $\Delta V$ near the poles of eq. (16).

Comparison of sensitivity data for contacts with different values of $V_{\mathrm{c}}$ and $R$ shows that the dependence of $S$ on these parameters (other things being equal) is in agreement with the theoretical analysis (eq. 17).

The frequency dependence of the response was measured for point contacts with the maximum values attained for $V_{\mathrm{c}}$ and $R\left(V_{\mathrm{c}}=0.7\right.$ to $1.0 \mathrm{mV}$, $R=5$ to $15 \Omega$ ). These measurements were carried out using a far-infrared monochromator with an echellette grating and appropriate sets of filters in the frequency range from 5 to $30 \mathrm{~cm}^{-1}$. The noise of the detection system, referred to it's input, was near $10^{-9} \mathrm{~V} / \mathrm{Hz}^{1 / 2}$.

The spectral data for an $\mathrm{Nb}-\mathrm{Nb}$ point contact in the frequency range $8-18 \mathrm{~cm}^{-1}$ are shown in figure $3 a$. It is clearly seen that there is structure periodic in wavelength. This structure seems to be associated with resonance phenomena due to the contact geometry and environment. As a rule the shape of spectral curves obtained at $V<V_{\omega}$ was independent of the shape of VIC and contact voltage $V$. We succeeded in obtaining an overall spectral curve, having joined together curves obtained with different echellette gratings as shown in figure $3 b$.

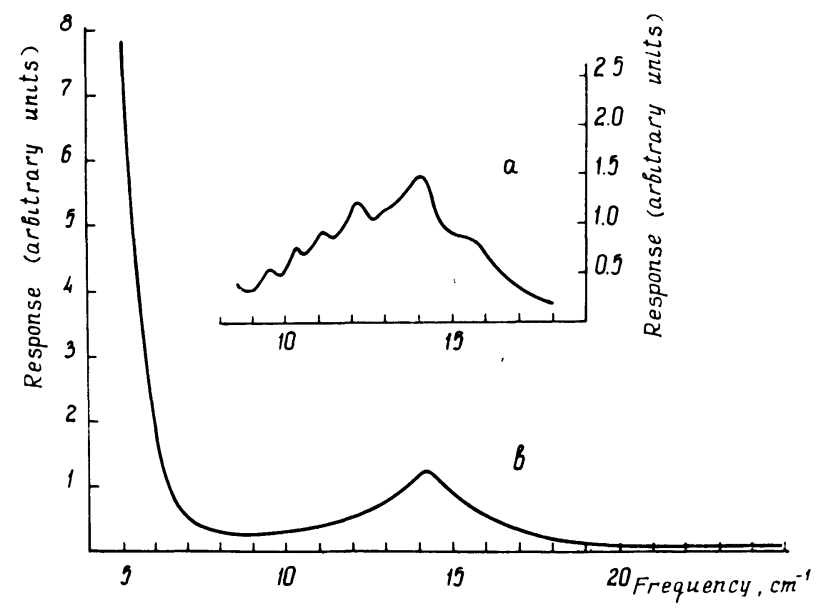

FIG. 3. - Sensitivity of the point contact as a function of frequency. a) range $8-18 \mathrm{~cm}^{-1} ; b$ ) range $5-25 \mathrm{~cm}^{-1}$.

Superconducting point contacts were found to be sensitive in the spectral range $5-30 \mathrm{~cm}^{-1}$. As shown in figure $3 b$, there is a maximum at $14 \mathrm{~cm}^{-1}$ and a rise of sensitivity at frequencies near $5 \mathrm{~cm}^{-1}$. Unfortunately our monochromator did not allow us to obtain radiation at frequencies lower than $5 \mathrm{~cm}^{-1}$, but estimates of sensitivity carried out after the experiments with another radiation sources show a peak near $\lambda \sim 2 \mathrm{~mm}$.

We have also measured the values of the voltage sensitivity and noise equivalent power referred to the power of radiation incident at the input of the 
light pipe. The following values were obtained at frequency $14 \mathrm{~cm}^{-1}(700 \mu \mathrm{m}): S \simeq 10^{3} \mathrm{~V} / \mathrm{W}$, $N E P \simeq 5 \times 10^{-12} \mathrm{~W} / \mathrm{Hz}^{1 / 2} ;$ at $5 \mathrm{~cm}^{-1}(2000 \mu):$ $S \simeq 10^{4} \mathrm{~V} / \mathrm{W}, N E P \simeq 5 \times 10^{-13} \mathrm{~W} / \mathrm{Hz}^{1 / 2}$. Theoretical estimates for the point contact with $R=5 \Omega$ at $\lambda=2 \mathrm{~mm}$ gives the values $S=10^{5} \mathrm{~V} / \mathrm{W}$,

$$
N E P=3 \times 10^{-15} \mathrm{~W} / \mathrm{Hz}^{1 / 2} \text {. }
$$

Experimentally obtained values of sensitivities are not yet equal to the theoretical limiting values. Decreasing of the noise fluctuation level to the selfnoise level and decreasing of transmittance losses will permit us to increase the sensitivity.

3. Hysteresis of the VIC. - Now let us consider some features of the using another type of weak link - a narrow superconducting bridge as the sensitive element of a microwave detector. One of the basic difficulties frequently encountered in this case relates to the hysteretic form of the VIC of the bridge. The hysteretic form of VIC is of little use for the purpose of detection of weak electromagnetic signals. We have carried out an experimental investigation of the hysteresis phenomenon and give an interpretation of the results obtained.

The measurements were taken with bridges obtained by evaporation $\mathrm{Sn}$ and $\mathrm{InSn}$ on a substrate made of glass, quartz or sapphire through a mask of special construction [14]. The width of the bridges was varied from 2 to $8 \mu \mathrm{m}$, the thickness from 500 to $3000 \AA$. The form of VIC and it's changes with temperature are qualitatively similar for bridges from $\mathrm{Sn}$ and from InSn. In figure 4 is shown a family of typical VIC for a dc current-driven bridge, with width $3.5 \mu \mathrm{m}$ and thickness $1200 \AA$ evaporated on a sapphire substrate. The critical current $I_{c}$ of such bridges depended on the temperature as $I_{\mathrm{c}}=\alpha_{0}\left(T_{\mathrm{c}}-T\right)^{3 / 2}$ for $T_{\mathrm{c}}-T<0.1 \mathrm{~K}$. When the temperature was reduced to $T_{\mathrm{c}}-T>0.1 \mathrm{~K}$ the form of this dependence became $I_{\mathrm{c}}=\gamma_{0}\left(T_{\mathrm{c}}-T-\delta\right)$, where $\delta$ is quantity about $0.01 \mathrm{~K}$. In temperature range where $T_{\mathrm{c}}-T$ was smaller than some quantity $\Delta T_{\mathrm{h}}=T_{\mathrm{c}}-T_{\mathrm{h}}$, the VIC is a simple curve (of the same form for increasing bias current, as for decreasing bias current), which is nonlinear and similar to a hyperbola for $I \gtrsim I_{\mathrm{c}}$ (see the curve at $T_{2}$ in Fig. 4). With reduction of the temperature to some $T_{\mathrm{h}}$ the VIC becomes double-valued when measured in the dc driven-current regime. In other words, hysteresis arises in the VIC (curve at $T_{3}$ ). The value of $\Delta T_{\mathrm{h}}$ depends on the size of the bridge (width and thickness of the constriction), on the material of the superconductor and the substrate. For example at the same conditions for Sn on a glass substrate $\Delta T_{\mathrm{h}}$ equals $0.03 \mathrm{~K}$, while on a sapphire substrate $\Delta T_{\mathrm{h}} \simeq 0.06 \mathrm{~K}$. With subsequent reduction of the temperature, the hysteresis grows.

All of this measurements were carried out with the scan frequency equal to $100 \mathrm{~Hz}$. In case of pulse measurements it was found that the form of the VIC

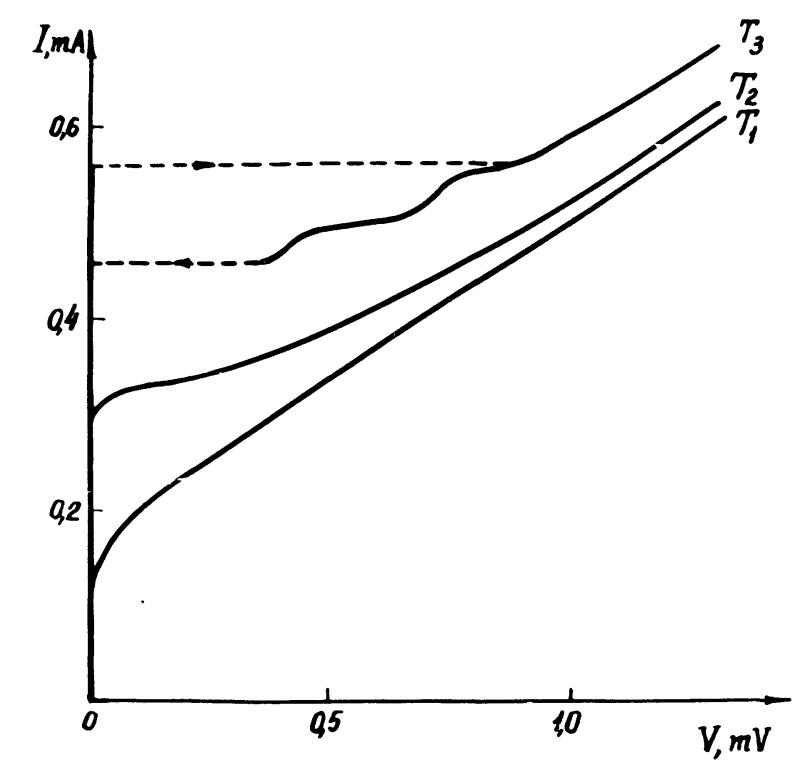

FIG. 4. - VIC of the superconducting InSn bridges. $T_{\mathrm{c}}=7.1 \mathrm{~K} . T_{1}>T_{2}>T_{3}$.

and its features practically did not change for pulse durations up to $1 \mu \mathrm{s}$. It was found also, that the form of VIC did not change qualitatively with a change of the aggregation condition of the ambient helium (liquid, gaseous or superfluid helium). The only difference was the effect that in liquid $\mathrm{He}$ at a temperature above the $\lambda$-point an irregular instability was observed on the reverse branche of the VIC. After the transition of $\lambda$-point and also in gaseous helium this instability disappeared. These facts indicate that the reason for the instability seems to be nucleate boiling of liquid helium near the constriction of the bridge.

The above enumerated experimental results testify to the important role, which heat generation plays in forming VIC's of narrow superconducting bridges. When the critical current $I_{\mathrm{c}}$ is exceeded an average voltage across superconducting bridge arises, dissipation of power takes place, heat generation begins and eventually this heat must be dissipated in the helium bath.

In the general case, there are two channels for heat flow out of the film : first, direct heat transfer from the surface of the film to the helium bath and, second, heat transfer through the substrate into the helium bath. In the case of the films evaporated on high heat conductivity substrates, the heat flow through the substrate prevails over the direct heat flow into the helium, because of the large contact area between the substrate and the helium. There is, however, an appreciable heat resistance at the metalsubstrate boundary. For example, the experimental value of heat transfer for the tin-quartz boundary accounts for about $1.6 \mathrm{~W} / \mathrm{cm}^{2} / \mathrm{K}$ [15]. Therefore the evaporation of the films even on high heat conductivity substrates cannot remove the effects of the overheating. 
By appealing to the processes of the heat generation, we can explain the presence of hysteresis in the VIC. For a quantitative description of the hysteresis phenomenon, we made use of the heat balance equation for the bridge :

$$
I^{2} R(I, T)=F S\left(T-T_{0}\right)
$$

where $S$ is the effective area of the heat sink into helium, $R(I, T)$ is the total resistance of the bridge, $F$ is an effective thermal conductivity, and $T_{0}$ is the helium bath temperature.

Then, determining the effective area, we used the temperature distribution, obtained in [18]. One can approximate the dependence $R(I, T)$ in the eq. (19), by the function :

$$
R(I, T)=0.5 R_{0}\left\{1+\tanh \left[\left(T-T_{\mathrm{c}}(I)\right) / \theta\right]\right\}
$$

where $\theta$ is the width of superconducting transition for the bridge, $R_{0}$ is the normal resistance, and $T_{\mathrm{c}}$ is the current-dependent critical temperature. From eq. (19) one can find the form of the VIC, which in general is a double-valued curve and it has two values of critical current, corresponding to the break-points (for dc current-driven bridge) at which $\mathrm{d} I / \mathrm{d} V=0$. For this purpose we solve eq. (19), combined with the next equation [17]

$$
I^{2}\left(\frac{\partial R}{\partial T}\right)_{I}=F S .
$$

The numerical solution shows, that this system has two roots $I_{\mathrm{c}}^{\prime}$ and $I_{\mathrm{c}}^{\prime \prime}$, which coincide at $T_{0} \geqslant T_{\mathrm{h}}$ and are distinct for $T_{0}<T_{\mathrm{h}}$, which signals the appearance of hysteresis. The solution of the system (19)-(20) is simplified in the temperature range $T_{\mathrm{c}}-T_{0}>0.1 \mathrm{~K}$, where according to experiment, the dependence of $I_{\mathrm{c}}$ on $T$ is linear. In this case

$$
\begin{aligned}
& I_{\mathrm{c}}^{\prime}=\gamma_{0}\left(T-\delta-T_{0}\right), \\
& I_{\mathrm{c}}^{\prime \prime}=\left(F S / R_{0}\right)\left(T_{\mathrm{c}}-T_{0}-\delta\right)^{1 / 2} .
\end{aligned}
$$

We can describe a measure of the development of hysteresis by the quantity $\beta=1-I_{\mathrm{c}}^{\prime \prime} / I_{\mathrm{c}}^{\prime}$. The calculations which were carried out showed, that the theoretical dependence of $\beta(T)$ was in a good agreement with the experiment [18]. Solving the system (19)-(20) under the condition $I_{c}^{\prime}=I_{c}^{\prime \prime}$ we can determine the temperature of appearance of the hysteresis $T_{\mathrm{h}}$. We obtained $\Delta T_{\mathrm{h}}=\left(\begin{array}{lll}2 & \theta F S / R_{0} & \alpha_{0}^{2}\end{array}\right)^{1 / 3}$. This result was obtained by the substitution in $R(I, T)$ the dependence $T_{\mathrm{c}}(I)=T_{\mathrm{c}_{0}}-\left(I / \alpha_{0}\right)^{2 / 3}$, which is valid for the temperature range $T_{\mathrm{c}}-T<0.1 \mathrm{~K}$, where $T_{\mathrm{c}_{0}}-$ the critical temperature at $I=0$. The choice of the correct dependence was conditioned by the fact, that the substitution of either experimental dependence of $I_{\mathrm{c}}$ on $T$ at our conditions yields $\Delta T_{\mathrm{h}}<0.1 \mathrm{~K}$. For tin bridge on a sapphire substrate we find $\Delta T_{\mathrm{h}}=0.055 \mathrm{~K}$, which coincides with the experimental value $\Delta T_{\mathrm{h}}=0.055 \mathrm{~K}$.

We also evaluated the time constant for the change of the film temperature and found it to be in the range from 0.01 to $0.1 \mu \mathrm{s}$. For this reason we did not find a difference in the form of the VIC when using the pulse measurement technique up to $1 \mu$ s.

Thus, to use a narrow superconducting bridge as a radiation detector, the VIC must not have hysteresis, and accordingly it is necessary to maintain the temperature of the bridge within range from $T_{\mathrm{c}}$ to $T_{\mathrm{h}}$. By improving the heat sink of the bridge one can expand this range of temperature (i. e. $T_{h}$ can be reduced).

\section{References}

[1] Grimes, C. C., Richards, P., Shapiro, S., J. Appl. Phys. 39 (1968) 3905.

[2] Kanter, H., Vernon, F. L., Phys. Lett. 35A (1971) 349.

[3] Blaney, T. G., Phys. Lett. 37A (1971) 19.

[4] Richards, P. L., Sterling, S. A., Appl. Phys. Lett. 14 (1969) 39.

[5] Grimes, C. C., Shapiro, S., Phys. Rev. 169 (1968) 397.

[6] LoNGACRe, A., Electronics 44 (1971) 44.

Licharev, K. K., Vestn, Mosc. Un-ta, Ser. PhysicaAstronomia 5 (1968) 104.

Russer, P., Arch. electr. Ubertrag. 23 (1969) 417.

[7] Vistavkin, A. N., Gubankov, V. N., Leschenko, G. F., Licharev, K. K., Migulin, V. V., Radiotechnikaelectronika 15 (1970) 2404.

[8] Aslamazov, L. G., Larkin, A. I., Piśma Zh. Eksp. \& Teor. Fiz. 9 (1969) 150.
[9] Volkov, A. F., YA, NAD'. F., Piśma Zh. Eksp. \& Teor. Fiz. 11 (1970) 92.

[10] Silver, A. H., Zimmerman, J., Appl. Phys. Lett. 10 (1967) 142.

[11] NAD', F. YA, Radiotechnika-electronika 17 (1972) 2360.

[12] Kanter, H., Vernon, F. L., Phys. Rev. Lett. 25 (1970) 588.

[13] Licharev, K. K., Semenov, V. K., Radiotechnika-electronika 16 (1971) 2167.

[14] Nad', F. Ya, PolyanskiI, O. Yu., Pribori $i$ technika eksperimenta 6 (1970) 210.

[15] Dorey, A. P., Cryogenics 5 (1965) 146.

[16] Volkov, A. F., Radiotechnika-electronika 17 (1972) 2581

[17] Bremer, I. W., Newhouse, V. L., Phys. Rev. 116 (1969) 309.

[18] Nad', F. Ya., PolyanskiI, O. Yu., " Electronica » 1972 deponent article. 